\title{
Structure and phase diagram of an adhesive colloidal dispersion under high pressure: A small angle neutron scattering, diffusing wave spectroscopy, and light scattering study
}

\author{
R. Vavrin, ${ }^{1, a)}$ J. Kohlbrecher, ${ }^{1}$ A. Wilk, ${ }^{1,2}$ M. Ratajczyk, ${ }^{2}$ M. P. Lettinga, ${ }^{3}$ J. Buitenhuis, ${ }^{3}$ \\ and G. Meier ${ }^{3}$ \\ ${ }^{1}$ Laboratory for Neutron Scattering, ETH Zurich and Paul Scherrer Institut, 5232 Villigen PSI, Switzerland \\ ${ }^{2}$ Institute of Physics, A. Mickiewicz University, Umultowska 85, 61-614 Poznan, Poland \\ ${ }^{3}$ IFF, weiche Materie, FZ-Jülich, Postfach 1913, 52428 Jülich, Germany
}

(Received 5 December 2008; accepted 22 February 2009; published online 20 April 2009)

\begin{abstract}
We have applied small angle neutron scattering (SANS), diffusing wave spectroscopy (DWS), and dynamic light scattering (DLS) to investigate the phase diagram of a sterically stabilized colloidal system consisting of octadecyl grafted silica particles dispersed in toluene. This system is known to exhibit gas-liquid phase separation and percolation, depending on temperature $T$, pressure $P$, and concentration $\varphi$. We have determined by DLS the pressure dependence of the coexistence temperature and the spinodal temperature to be $d P / d T=77 \mathrm{bar} / \mathrm{K}$. The gel line or percolation limit was measured by DWS under high pressure using the condition that the system became nonergodic when crossing it and we determined the coexistence line at higher volume fractions from the DWS limit of turbid samples. From SANS measurements we determined the stickiness parameter $\tau_{B}(P, T, \varphi)$ of the Baxter model, characterizing a polydisperse adhesive hard sphere, using a global fit routine on all curves in the homogenous regime at various temperatures, pressures, and concentrations. The phase coexistence and percolation line as predicted from $\tau_{B}(P, T, \varphi)$ correspond with the determinations by DWS and were used to construct an experimental phase diagram for a polydisperse sticky hard sphere model system. A comparison with theory shows good agreement especially concerning the predictions for the percolation threshold. From the analysis of the forward scattering we find a critical scaling law for the susceptibility corresponding to mean field behavior. This finding is also supported by the critical scaling properties of the collective diffusion.
\end{abstract}

(C) 2009 American Institute of Physics. [DOI: 10.1063/1.3103245]

\section{INTRODUCTION}

In this paper we are concerned with the structure and phase diagram of a polydisperse adhesive hard sphere system. ${ }^{1-7}$ This system is known to display a complex phase behavior. Its phase diagram not only shows a gas-liquid phase separation line but also a percolation or gel line, which intersects the coexistence line. Numerous studies have been performed trying to shed a light on the structural properties that underlie this phase behavior. These studies can be roughly divided into real space studies, using microscopical techniques, probing the real space by exploiting the typical colloidal size ${ }^{8-10}$ rheological techniques, ${ }^{11,12}$ and scattering techniques, ${ }^{13-15}$ probing the reciprocal space. Some of these studies accumulated recently in the suggestive picture that the formation of percolated networks is directly connected to spinodal-like phase separation, ${ }^{8}$ although, as we will discuss in this paper, also other scenarios are conceivable.

The system of sticky hard colloidal spheres belongs to a class of systems where phase separation competes with arrested states like gels or glasses. ${ }^{11}$ Systems where these kind of effects are at hand vary between model colloidal systems such as highly concentrated hard spheres with added deple-

${ }^{a)}$ Electronic mail: ronny.vavrin@psi.ch. tion interaction, ${ }^{16}$ to biological systems ${ }^{12}$ to networks of rods. ${ }^{17}$ Thus, identifying the structural features underlying such a complex phase behavior for a system of sticky hard spheres would not only be of interest for the colloid field but would also shed light on general physical aspects which combine glass physics (and its most prominent theoretical approach, the mode coupling theory) with colloidal physics and gel phases and demixing phenomena. Thus, knowledge of the connection between gelation and phase separation for sticky hard spheres would greatly help also the understanding of other arrested systems.

The model system we use in this paper, and which was used in our preceding paper, ${ }^{18}$ consists of silica spheres grafted with octadecyl chains in toluene at various volume fractions. The reason to use exactly this system of sticky hard sphere system as a model system is twofold. First, the interaction between the particles, and therefore the phase behavior, can very easily be tuned varying the temperature, as toluene is a marginal solvent for the grafted polymers. This has been elucidated by various experimental techniques. ${ }^{19-29}$ The molecular background of this interaction was found by Roke et al. ${ }^{27,28}$ More interestingly, as we will show in this paper, the interaction can equally be tuned with pressure such that a liquid-liquid phase transition can be induced also by changing the pressure. ${ }^{30}$ Second, sticky hard spheres can 
be theoretically very elegantly modeled within the framework of the Percus-Yevick approximation of hard spheres with a square well attractive potential, provided that a certain limit is taken in which the range of the well becomes zero and its depth infinite such that the second virial coefficient remains finite. This model, named after its inventor Baxter, ${ }^{31}$ is capable to map the interactions between colloidal particles as long as the interaction range is small compared to the particle size. The connection to the experimentally measured values such as the structure factor can be readily made with only one parameter, namely, the stickiness parameter $\tau_{B}$, which is a control parameter within a square well attractive potential, that can be thought of a dimensionless temperature (see Sec. II). The stickiness $\tau_{B}$ measures the change from hard sphere behavior (large $\tau_{B}$ ) to sticky behavior (small $\tau_{B}$ ). The only other parameter that needs to be accounted for to make the link to the experiments is the polydispersity, for which quite recently a numerical algorithm for the structure factor within the Percus-Yevick approximation was developed, called the Robertus model. ${ }^{32}$ The clue is now that the full phase diagram, including phase coexisting lines and percolation lines, can be predicted knowing how the stickiness parameter $\tau_{B}$ depends on temperature or pressure. ${ }^{29}$

This leads us to the goal of this paper. What we set out to do is to get this pressure and temperature dependence of $\tau_{B}$ in the part of the phase diagram where there is one single ergodic phase. For this we determine the structure factor with small angle neutron scattering (SANS) throughout phase space, i.e., for different concentrations, temperatures, and pressures. We fit all this data with one single global fitting approach which fits all parameters of all measured curves simultaneously, using the Robertus model. Already in a previous paper we showed that for the same system at high dilution this fit routine could provide all trivial parameters such as the contrast and the form factor at different pressures. ${ }^{18,33}$ Probing now the full single phase space we obtain an unambiguous hold on the temperature and pressure dependence of $\tau_{B}$, and therefore also on the location of the percolation and coexistence line. Then we actually determine these lines by a combination of experiments: we use the dynamic method, diffusing wave spectroscopy (DWS), to correlate the ergodic to non ergodic transition with exactly this percolation transition after applying high pressure, which has not been reported so far. The liquid-liquid phase transition or coexistence line (in the language of phase separation that is also called the binodal curve) can be determined by simple visual inspection or by measuring the turbidity. The pressure derivative of the transition temperature was determined by dynamic light scattering (DLS) for a volume fraction around $\varphi=0.1$ in order to compare our system parameters with those already published ${ }^{30}$ (in the order of $77 \mathrm{bar} / \mathrm{K}$ ). The expected phase diagram will shift with pressure according to this value. We sketch that in Fig. 1. We will show that the phase diagram as calculated with the most recent theoretical approach for the structure factor using the experimentally obtained $\tau_{B}(P, T)$ as an input nicely coincides with our independently experimentally determined phase diagram. As such, this is the first self-consistent set of data, where the

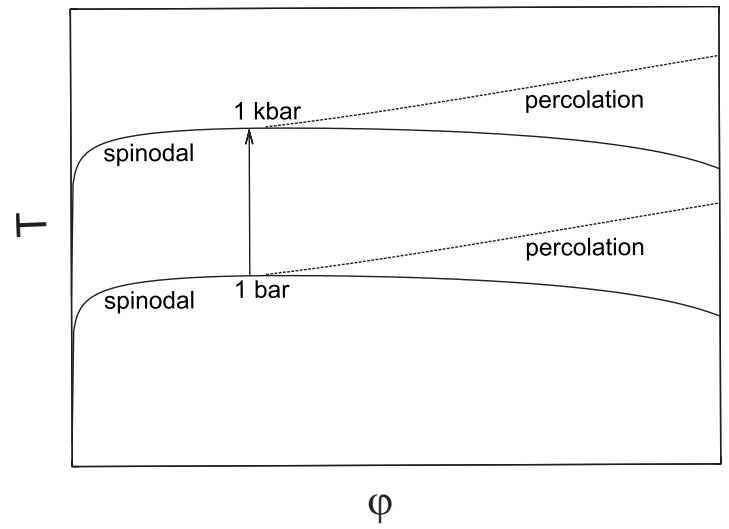

FIG. 1. Schematic phase diagram of adhesive hard sphere system. Shown is the action of pressure on the loci of coexistence and percolation lines. The observed temperature shift under the action of $1 \mathrm{kbar}$ pressure amounts to about $\Delta T=13 \mathrm{~K}$.

phase behavior can be predicted and tested using experimentally obtained molecular input of the interaction potential between the particles.

The role of the pressure here is that it is a very clean way to access points in phase space because changing pressure renders new points that are homogeneous in pressure and temperature. Moreover, the point can be accessed with high precision. We will show that this is important when approaching the critical point, being on the low or high concentration side of the percolation line, because then the scaling of the critical structure can be tested. Here we also take advantage of testing reciprocal space, because this is the most accurate way to get an averaged parameter like the critical structure. Also a very interesting feature is, however, that points in phase space can be accessed within seconds or faster. This enables us to access the kinetics of phase separation or of the percolation and as such it gives a handle on study of the interplay between phase separation and percolation, which is still a puzzle. As such, this paper reports also a feasibility study for such experiments.

This paper is organized as follows: In Sec. II we will present a theoretical sketch on the pressure and temperature dependent interactions in sticky hard sphere systems, in Sec. III we will present the experimental methods used: DLS, DWS, and SANS. In Sec. IV, we will present the results and discuss them in five subsections: (Sec. IV A) for the determination of the pressure dependence of the transition temperatures, (Sec. IV B) the DWS results for the determination of the gel line, in Sec. IV C, results from the global fit of the SANS data. In Sec. IV D we will discuss the critical scaling laws and in Sec. IV E, we present the experimental phase diagram and compare it to the theoretical one. We conclude with Sec. V.

\section{THEORETICAL: PRESSURE AND TEMPERATURE DEPENDENCE OF INTERACTIONS IN STICKY SPHERE SYSTEMS}

Adhesive polydisperse core-shell particles are assumed to interact on the pair level through a interaction potential given by the Baxter model. ${ }^{21,31}$ This pair-interaction potential is a square well of infinitesimal width and infinite depth 
which is superimposed on a hard core repulsion. The pairinteraction potential between the colloids has the form

$$
\begin{aligned}
& V_{B}(r) \\
& =\lim _{\Delta \rightarrow 0} \begin{cases}\infty & r<2 R, \\
k_{B} T \ln \left[12 \tau_{B}\left(\frac{\Delta}{2 R+\Delta}\right)\right] \text { for } \quad & 2 R \leq r \leq 2 R+\Delta, \\
0 & r>2 R+\Delta,\end{cases}
\end{aligned}
$$

where $R$ is the hard core radius of the colloid and $\Delta$ is the width of the square well interaction potential. For small widths of $\Delta$, being a measure of the length of the grafted chains, the scattering behavior only depends on the stickiness parameter $\tau_{B}$, or attraction parameter, which is a function of both temperature and pressure. $\tau_{B}$ is often referred to as a dimensionless quasitemperature. To relate the stickiness parameter $\tau_{B}$ to the real temperature $T$, the model from FloryKrigbaum, frequently used in describing the interaction between polymer chain segments, is used. It relates the depth $\varepsilon_{D}$ of a square well potential $V_{\mathrm{SW}}(r)$

$$
V_{\mathrm{SW}}(r)= \begin{cases}\infty, & r<2 R, \\ -\varepsilon_{D}, & 2 R \leq r \leq 2 R+\Delta, \\ 0, & r>2 R+\Delta,\end{cases}
$$

to the temperature $T$ by assuming that the depth $\varepsilon_{D}$ of the square well potential depends linearly on the parameter $L$, which depends on the overlap volume of the two spheres and the difference between the $\Theta$-temperature and temperature $T$. The $\Theta$-temperature in this model is a measure of the enthalpic and entropic interactions between solvent and solute.

$$
\varepsilon_{D}= \begin{cases}L(\Theta-T) k_{B}, & T<\Theta, \\ 0, & T \geq \Theta .\end{cases}
$$

The $\Theta$-temperature is assumed to vary in first approximation linearly with $P$ according to

$$
\Theta(P)=\Theta_{0}+\frac{d \Theta}{d P}\left(P-P_{0}\right)
$$

Here, the new term $d \Theta / d P$ appears which is proportional to the compressibility. We have measured that the volume change in the particles while applying pressure is small, ${ }^{33}$ in the order of $10^{-5}$, and hence comparable to compressibilities found in polymeric systems from the pressure dependence of the Flory-Huggins interaction parameter $\chi$, being proportional to $\Theta .{ }^{34}$ Clearly, the depth $\varepsilon_{D}$ of the interaction potential determines the phase diagram and thus also the pressure as it has the linear pressure dependence of the theta temperature and $d P / d \Theta=$ const. This is shown schematically in Fig. 1.

These two potentials, Eqs. (1) and (2), are equivalent and can be equated to solve for $\tau_{B}$. The result is

$$
\frac{1}{\tau_{B}}=\frac{12 \Delta}{2 R+\Delta} \exp \left[\frac{\varepsilon_{D}}{k_{B} T}\right]=\frac{12 \Delta}{2 R+\Delta} \exp \left[L\left(\frac{\Theta(P)}{T}-1\right)\right] .
$$

This expression allows now to correlate the degree of interaction $\tau_{B}$ (with $T, P$ as parameters) with details of the potential: $R$ and $\Delta$. As already shown in literature ${ }^{21,31}$ the exact shape of the interaction potential is not significant as long as the width of the attractive well is much smaller than the particle diameter, i.e., $\Delta \ll 2 R$.

At $P=1$ bar, $\Theta(P)$ is equal to $\Theta_{0}$, and Eq. (5) can be rewritten to give $T$ as a function of $\tau_{B}$,

$$
T=\frac{\Theta_{0} L}{\left.-\ln \left(\frac{12 \Delta \tau_{B, P=1 \mathrm{bar}}}{2 R+\Delta}\right)+L\right]} .
$$

This relation is used to convert $\tau_{B}$ into $\mathrm{T}$ provided all other parameters are known.

The knowledge of the theoretical phase diagram for an adhesive hard sphere (AHS) system has been a subject of a long discussion. We refer to the recent numerical calculations of Miller and Frenkel ${ }^{7}$ and to the most recent calculations by Fantoni et al. ${ }^{35}$ These attempts circumvent the deficiencies which were present in the oversimplified model by Penders and $\mathrm{Vrij}^{21}$ to calculate the spinodal. To cite Miller and Frenkel: "A major obstacle in the application of the adhesive hard sphere model to the description of experimental data is that the model's phase behavior is only known through the approximate and conflicting theoretical results of Percus-Yevick theory." However, in the oversimplified model a rather simple analytical expression can be given to relate the stickiness to the volume fraction. This expression reads

$$
\begin{aligned}
& \tau_{B}=\frac{\varphi}{1-\varphi}\left(\sqrt{\frac{1+\varphi / 2}{3 \varphi}}-1\right), \quad \varphi<0.12, \\
& \tau_{B}=\frac{1+4 \varphi-14 \varphi^{2}}{12(2 \varphi+1)(1-\varphi)}, \quad \varphi \geq 0.12 .
\end{aligned}
$$

The model gives for the critical concentration $\varphi_{c}$ $=0.1213$ at a stickiness $\tau_{B}=0.0976$. However, the simulation results by Miller and Frenkel ${ }^{7}$ determined the critical point at $\varphi_{c}=0.266$ at $\tau_{B}=0.1133$. This is supported by Fantoni et al. ${ }^{35}$ The theoretical phase diagram for an AHS system is shown in Fig. 2. Note that the differences in $T$ reflecting the disparity in $\tau$ from both models is in the order of $0.4 \mathrm{~K}$, moreover the coexistence line is rather flat within our volume fraction range. Hence, from an experimental point of view, the differentiation between these various theories seems to be difficult. Likewise the differences between the refined models ${ }^{7,35}$ and the simple $\mathrm{C} 1$ model, as referred to Fantoni et al. $^{35}$ is also small for $\varphi$ smaller than $0.1-0.15$. The $\mathrm{C} 1$ model is to a good approximation given by Eq. (7).

Further, it is known theoretically ${ }^{3}$ and also confirmed by simulations and experiments ${ }^{5,6,24}$ that in sticky hard sphere systems percolation (kinetic transition) also occurs beneath the liquid-liquid phase transition. The dynamic percolation line is obtained on the basis of an Ornstein-Zernike equation 


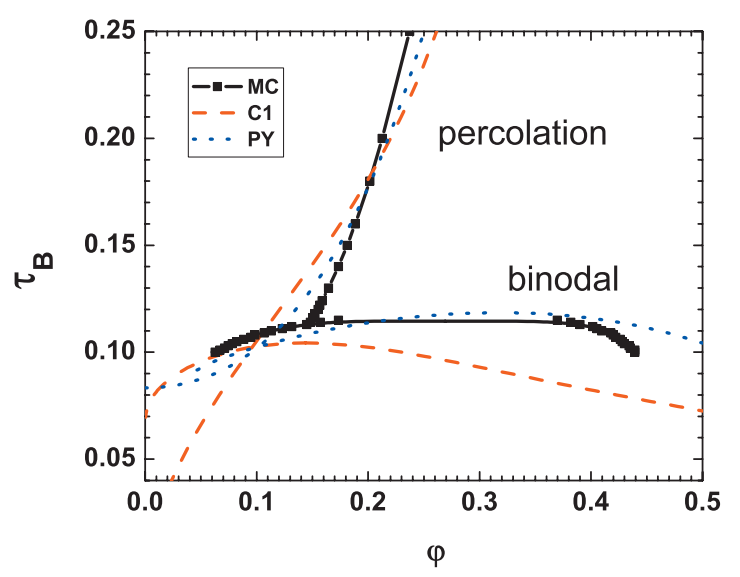

FIG. 2. (Color) Theoretical phase diagram of an adhesive hard sphere model taken from Fantoni et al. (Ref. 35. Their new data (dotted lines) is denoted as PY and is in this plot compared to MC simulations from Miller and Frenkel (Ref. 7) which are shown as full lines and filled squares. Dashed lines denote the model $\mathrm{C} 1$ according to Fantoni et al. (Ref. 35). In this model, the value for $\varphi_{c}$ is to a good approximation given by Eq. (7).

in the Perkus-Yevick approximation. In this calculation the so-called pair connectedness function is utilized, which relates the probability of simultaneously finding a particle in the same cluster at a given distance. The stickiness $\tau_{B}$, at which the system percolates, given in terms of volume fraction, reads ${ }^{3}$

$$
\tau_{B}=\frac{19 \varphi^{2}-2 \varphi+1}{12(1-\varphi)^{2}} .
$$

The resulting curve shows a crossing point with the spinodal line at about a volume fraction of $\varphi \sim 0.07$ in the Penders and Vrij model. ${ }^{21}$ However, the recent simulations ${ }^{7,35}$ show that the intersection between coexistence and percolation line occurs at some higher volume fractions at about $12 \%$ volume fraction. For $\varphi<0.12$ it is found experimentally that the percolation lies below the coexistence. For larger volume fractions, the percolation is always above the coexistence. The physical interpretation of the region between the spinodal and the percolation intersection is under debate, since some authors claim ${ }^{12,36,37}$ that here the nucleation is hindered or frustrated and in a sense the differentiation between percolation and nucleation is difficult to make experimentally. It should be noted that in the calculations by Miller and Frenkel ${ }^{7}$ polydispersity corrections have not been implemented. Nevertheless, the value for the critical density is shifted toward higher values as compared to the calculation along Eq. (7). However, it is known experimentally that the phase diagram of polydisperse systems differs considerably from its monodisperse analog at least for polymeric systems. ${ }^{38}$ We see no argument why that should be different for colloidal mixtures. Taking the polydispersity into account, ${ }^{35}$ the value for the critical density seems to be even higher (cf. Fig. 2). Clearly, from the simulations in comparison to simple calculations the critical point is found at much higher volume fractions.

We intend to put our experimental results as quantitative as possible into this theoretical phase diagram, thereby performing and presenting experiments, which have so far not been applied to these systems. We will put much emphasis on the $\varphi=0.16$ sample, since there the interplay between percolation and coexistence can be studied best.

\section{EXPERIMENTAL}

\section{A. Materials}

Silica core particles were prepared according to Stöber et $a l^{39}$ and then grafted with stearyl alcohol following van Helden $^{40}$ to obtain octadecyl chains chemically anchored onto the surface. All samples were mixed from two concentrated colloidal dispersions of the particles; one in fully deuterated and one in fully protonated toluene. The analysis of the same particles as shown in our previous paper ${ }^{18}$ gave for the core diameter $R_{c}=32.3 \mathrm{~nm}$, for the shell thickness (grafted layer thickness) $\Delta R=2.3 \mathrm{~nm}$ and for the size polydispersity $\sigma$, assuming a Schulz-Zimm distribution of the overall radius, $\sigma=0.124$.

\section{B. Light scattering}

Light scattering was measured either in the SANS high pressure cell at PSI (Ref. 41) or in the Jülich high pressure cell. ${ }^{42}$ The intensity time-correlation function $G^{(2)}(q, t)$ was calculated in real time by means of a Flex99OEM-12 digital correlator (correlator.com, USA) or an ALV 5000E digital correlator (ALV GmbH, Langen, Germany). For the homodyne case the intensity autocorrelation function $G^{(2)}(q, t)$ $=\langle I(q, t) I(q, 0)\rangle$ is related to the electric field, $E(q, t)$, through the normalized autocorrelation function $g^{(1)}(q, t)$ via the Siegert relation ${ }^{43}$

$$
G^{(2)}(q, t)=\langle I(q)\rangle^{2}\left(1+f\left|g^{(1)}(q, t)\right|^{2}\right),
$$

where $f$ is an experimental factor and $\langle I(q)\rangle$ is the mean intensity (also named static intensity), and $q$ $=(4 \pi n / \lambda) \sin (\theta / 2)$ is the length of the scattering vector, $n$ is the refractive index of the scattering medium, $\theta$ is the scattering angle, and $\lambda$ is the wavelength of the incident light in vacuum. The field autocorrelation function $g^{(1)}(q, t)$ is related in the simplest case to a diffusion coefficient $D$ of the system with equilibrium concentration fluctuations via $g^{(1)}(q, t)=\exp \left(-D q^{2} t\right)=\exp \left(-\left(q^{2} / 6\right)\left\langle\Delta r^{2}(t)\right\rangle\right)$ with $\left\langle\Delta r^{2}(t)\right\rangle$ $=6 D t$ being the mean square displacement. Then $\ln g^{(1)}(q, t)$ is linear in $t$, hence $\ln g^{(1)}(q, t)=-\Gamma t=-t / \tau$, where $\Gamma$ denotes the relaxation rate and $\tau$ is the relaxation time, with $\Gamma=D q^{2}$. To model the field autocorrelation function $g^{(1)}(q, t)$ in the case of a distribution of diffusion coefficients or other phenomena which lead to a deviation of a single exponential decay $\left[\ln g^{(1)}(q, t)\right.$ versus $t$ is not linear], we used either a so-called cumulant expansion or the well established CONTIN algorithm which is used in analyzing dynamic light scattering data. ${ }^{44}$ The cumulant expansion assumes for the normalized electric field autocorrelation function $g^{(1)}(q, t)=\exp \left(-\Gamma t+\left(\mu_{2} / 2 !\right) t^{2}-\cdots\right)$, where $\mu_{2}$ is the second order cumulant and is a measure of the variance of the distribution. Clearly, for a single exponential decay, $\mu_{2}$ vanishes and the simple relation between the rate and decay time emerges. However, in some cases it is better to fit with the full expression and allow for a small correction in $\Gamma$ due to a nonzero $\mu_{2}$. We use this algorithm for the DWS data reduc- 
tion mostly. However, the DLS data is analyzed using CONTIN, which gives access to the distribution of relaxation times $L(\tau)$ via $^{44}$

$$
g^{(1)}(t)=\int_{0}^{\infty} \exp \left(-\frac{t}{\tau}\right) L(\tau) d \tau
$$

and thus to the distribution of diffusion coefficients and concurrently to the size distribution of the particles since $D$ is related to size via a Stokes-Einstein relation $D$ $=(k T /(6 \pi \eta r))$ with $\eta$ being the solvent viscosity and $r$ the particle radius, $k T$ has the usual meaning. Using this set of ingredients we have determined $G^{(2)}(t)$ as a function of temperature and pressure for a fixed scattering angle.

\section{Diffusing wave spectroscopy}

Light scattering methods are difficult to apply to turbid samples since most methods use a single scattering approach. They therefore have to suppress multiply scattered light either with technical tricks or an elaborate analysis. Contrary to this, the DWS technique can take advantage of the multiple scattering in highly turbid samples. When a sample is turbid enough to totally randomize the scattering direction of a photon, the photon path can be approached as a diffusion process with the according statistical models. The correlation function of the scattered light then reflects the (heavily averaged) dynamics of the sample and is given for transmission geometry by

$$
g^{(1)}(t)=\exp \left(-\frac{k_{0}^{2}\left(\frac{L}{l^{*}}\right)^{2}}{6}\left\langle\Delta r^{2}(t)\right\rangle\right),
$$

where $k_{0}$ is given by $2 \pi n / \lambda, L$ is the sample thickness and $l^{*}$ stands for the transport mean free path. With this simplified version of the formula derived in Ref. 45 it is possible to measure the mean square displacement inside a turbid sample, which then, for example, can be used to estimate the average particle size of a colloidal system. How to treat not perfectly turbid samples is discussed in Sec. IV.

The DWS technique cannot only be used to measure the diffusion coefficient of particles in a turbid sample but it can also quite easily be enhanced such as to characterize samples in dynamically arrested states like a glass phase or a gel. The characterization of nonergodic samples is only possible through a proper ensemble average, as averaging over time represents only a limited part of the sample. A simple way to measure the ensemble average is the so-called two-cell technique, ${ }^{46}$ where the signal from the sample is led through another (turbid) scattering medium, the "second cell." The second cell ensures the proper averaging of the sample signal and features an ergodic decay which is slower than the one of the sample and therefore guarantees a physically correct baseline without distortion of the sample signal. When both cells scatter independently i.e., no photons are scattered loop like back and forth between the two cells, the correlation function of the sample $G^{(2)}(t$, sample) can be extracted from the measured correlation function $G^{(2)}(t)$ through division by the separately measured correlation function $G^{(2)}(t$, scndcell) of the second cell only:

$$
G^{(2)}(t, \text { sample })-1=\frac{G^{(2)}(t)-1}{G^{(2)}(t, \text { scndcell })-1} .
$$

In the home built setup at PSI, we use a rotating depolished glass as second cell, whose correlation function decay time can easily be varied through the rotation speed.

\section{Small angle neutron scattering}

The raw SANS data that we have measured with the high pressure cell setup were analyzed using the BERSANS software package, ${ }^{47}$ which is a standard program to analyze SANS data. The scattering cross section $(d \Sigma / d \Omega)(q)$ is related to physical properties of our system under study via ${ }^{48}$

$$
I(q)=\frac{d \Sigma}{d \Omega}(q)=c \frac{N_{A}}{M_{W}}\left(b-V \rho_{0}\right)^{2}\left\langle f^{2}(q)\right\rangle S(q),
$$

where $\left\langle f^{2}(q)\right\rangle$ is the size averaged squared form amplitude [commonly known as form factor $P(q)$ ] for spherical shells ${ }^{18}$ which is normalized to 1 for $q=0$ and $S(q)$ is the average static structure factor, which has been calculated according to Robertus et al. $^{32}$ which takes into account the inter-particle correlations. Only for sufficiently diluted systems $S(q)=1$ for all $q, b$ is the scattering amplitude (scattering length) of the colloid, $V$ is its volume, and $\rho_{0}$ is the scattering length density of the solvent. $c=\left(N M_{W} / V_{\text {tot }} N_{A}\right)$ is the particle concentration in $\mathrm{g} / \mathrm{cm}^{3}$ with $V_{\text {tot }}$ being the illuminated sample volume, $N$ is the number of particles in the illuminated volume, $N_{A}$ is the Avogadro number, and $M_{W}$ is the molar mass.

We had performed SANS scattering experiments ${ }^{18}$ at a volume fraction of $\varphi=2 \times 10^{-3}$ to determine the form factor $\left\langle f^{2}(q)\right\rangle$. For our core-shell particles the form factor is known analytically. In that study we had measured SANS at volume fractions of $5 \%, 11.2 \%, 16 \%$, and $39.2 \%$. For all these volume fractions we observed a structure factor. A quantitative analysis in terms of a sticky hard sphere structure factor for polydisperse systems using a numerical algorithm given by Robertus et al. ${ }^{32}$ had been carried out on the basis of the Baxter model, which we showed in our preceding paper, ${ }^{18}$ where only the volume fraction and the scattering contrast had been varied. Here in this paper we go one step further by introducing a stickiness parameter, which is a measure of the interactions in our colloidal system. The stickiness $\tau_{B}$ is determined applying a global fit routine which simultaneously fits all scattering curves taking into account SANS scattering curves at all volume fractions at various temperatures and pressures in the homogeneous regime. This procedure allows extracting reliably the parameters of the Baxter model and will be shown in Sec. IV C.

\section{RESULTS AND DISCUSSION}

\section{A. Determination of $d P / d T_{\text {trans }}$ and spinodal-binodal using DLS}

We have measured with light scattering the pressure dependence of various transition temperatures (the coexistence or binodal and the spinodal temperature), i.e., $d P / d T$. This is absolutely essential in our experiments, since the magnitude of its values will influence the experimental accessibility of a 

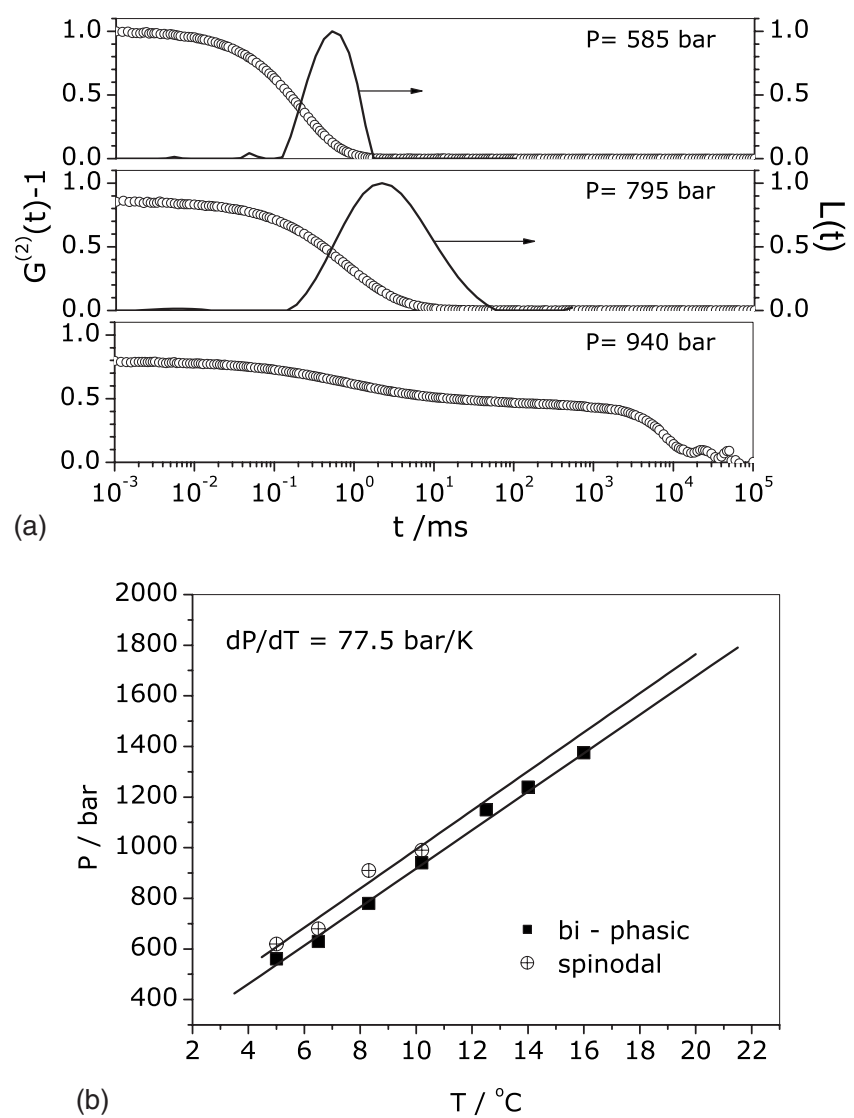

FIG. 3. (a) The transition is determined by the pressure at which the correlation functions clearly deviate from a narrowly distributed CONTIN distribution. Here as an example measurements at $T=8.3{ }^{\circ} \mathrm{C}$. The single exponential decay gives a narrow CONTIN peak, top at $P=585$ bar, and broadening indicating crossing the coexistence, middle at $P=795$ bar. Correlation function at the bottom at $P=940$ bar indicates a transition to the spinodal region. No CONTIN analysis possible. (b) Phase transition pressures at a volume fraction of $\varphi=0.10$ as a function of temperature. The two straight lines in the figure have a slope of $d P / d T_{\text {trans }}=77.5 \pm 2.5 \mathrm{bar} / \mathrm{K}$ as determined by linear fits to the data.

variety of physically interesting phenomena and further defines the necessity of using pressure as a variable. The experiments were performed with a $\varphi=0.1$ solution using a light scattering setup for high pressure which is described elsewhere. ${ }^{42}$

In Fig. 3(a), top, we show an example for the shape of the correlation function obtained from a homogeneous sample at $P=585$ bar. The corresponding CONTIN analysis gives a narrow distribution in agreement with a single exponential decay of $g^{(1)}(q, t)$. Increasing the pressure to $P$ $=795$ bar leads to a broadening of the correlation function [cf. Fig. 3(a), middle] and is indicative for crossing the binodal (coexistence line). The additional slower processes at these elevated pressures are due to the formation of droplets having another composition and thus sufficient index of refraction difference to cause a broadening of the correlation function at longer times. Applying the CONTIN algorithm these small traces of nucleated particles can be straightforwardly detected, although the corresponding correlation functions differ not much in the vicinity of the transition as can be seen from inspecting Fig. 3(a) top and middle. A close comparison of these two correlation functions also reveals a small decrease in the contrast at higher pressure, which stems from a portion of elastic scattering from the phase boundaries acting as local oscillators and thus reducing the contrast. This effect is also indicative for crossing the binodal together with the broadening; however, it is not very marked.

Sticking to our chosen example at $T=8.3{ }^{\circ} \mathrm{C}$ we have further increased the pressure although the coexistence was already reached. For pressures up to 900 bar the situation is in accord with the findings already outlined: a broadening at about 750 bar indicating the crossing of the binodal. The situation became more complex at an even higher pressure (here $P=940$ bar), when again a strong change in shape of the correlation function was observed as is shown in Fig. 3 (a), bottom. This change is different from the one described in Fig. 3(a), middle, because the correlation function for $P$ $=940$ bar seems to stay on a level of about 0.35 and is forced to relax to zero only because the ALV correlator assumes a zero base line at long times. Nevertheless, in principle, we think that what we observe is indicative for either the spinodal or the percolated state of the system. Percolation, however, can be very likely ruled out for three reasons. (i) There is no time dependence in the experiment, since we find that the crossing of the binodal and likely spinodal is a reversible effect: It takes the system not much time to reach homogeneity after the pressure is released below the coexistence pressure and spinodal pressure and (ii) from our DWS data (following chapter) we find the percolation threshold from the ergodic to nonergodic transition at higher pressures than 940 bars. Last but not least, we know (iii) that from inspection of the experimental phase diagram shown in our previous paper that the gel line is very steep and the temperature difference at $10 \%$ volume fraction between binodal and gel line is about $4 \mathrm{~K}$. That would correspond to about 300 bar pressure difference and is hence incompatible with our findings.

In Fig. 3(b) we plot the loci of all transition pressures as function of temperature and find linear relations with a slope of $d P / d T=77.5 \mathrm{bar} / \mathrm{K}$ for the binodal line and the spinodal line. The extrapolation of the data to give the transition temperature at $P=1$ bar yields $T=(-2.5 \pm 0.75){ }^{\circ} \mathrm{C}$ for the binodal and $T=(-3.5 \pm 0.75){ }^{\circ} \mathrm{C}$ for the spinodal. Both values are close to each other and confirm within the experimental accuracy the result of the already published phase diagram based on a simple test tube experiment. ${ }^{18}$ The experimentally determined coefficient $d P / d T=77.5 \mathrm{bar} / \mathrm{K}$ is in agreement with the value reported by De Kruif and Schouten. ${ }^{30}$ They find the pressure dependence of the transition temperature at $\varphi=0.13$ to be $d P / d T_{\text {trans }}=77 \mathrm{bar} / \mathrm{K}$, although the phase transition temperature of our system differs about $9 \mathrm{~K}$ from their sample. The value for $d P / d T_{\text {trans }}=77.5 \mathrm{bar} / \mathrm{K}$ for the spinodal transition is identical to the one for the coexistence transition and has not been reported before. The value of the experimentally determined derivative seems to be independent of $\varphi$, at least between $\varphi=10 \%-13 \%$, however, according to Fig. 2 variations of $\tau_{B}$ in the volume fraction range between $\varphi=5 \%$ and $16 \%$ are at most $10 \%$, which corresponds to about $0.3 \mathrm{~K}$ in temperature, cf. Fig. 7. Thus, this variation is far beyond our experimental resolution.

The so obtained phase boundaries are considered to be 

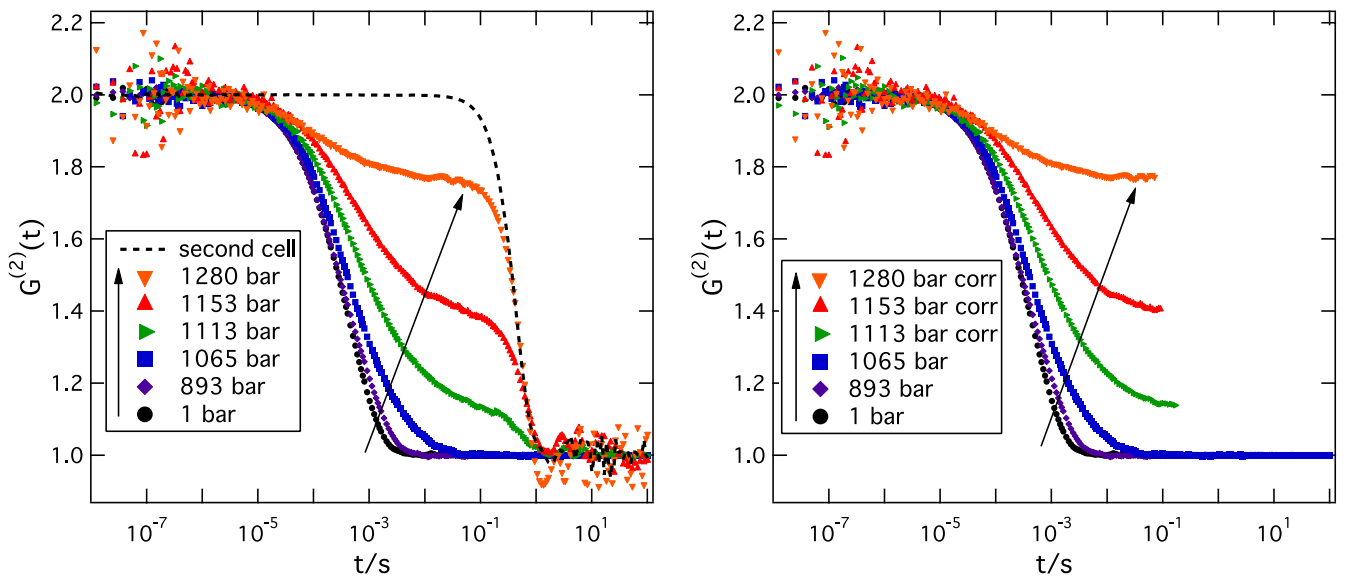

FIG. 4. (Color online) Normalized raw data of the two-cell DWS setup for a volume fraction of $16 \%$ is shown on the left side. On the right side, the same data are corrected for the decay of the second cell. With increasing pressure, the correlation functions decay at later lag times and eventually build up a plateau, which is a clear sign of a nonergodic state. Temperature of measurement is $T=14{ }^{\circ} \mathrm{C}$.

our reference states, because the data are clear, reproducible and confirming earlier results. In the following, we extend the pressure range, provide a dynamic analysis [cf. Fig. 3(a)] and present data obtained from several temperatures and volume fractions.

\section{B. Characterization of the gel line using DWS results}

In the last paragraph we determined the spinodal and binodal. Now we turn to the determination of the ergodic/ nonergodic transition. Since turbidity plays a role, a very convenient way to characterize turbid samples is to use the two-cell DWS technique described in Sec. III C. We applied it to a home built high pressure cell in Jülich ${ }^{42}$ which allows for measuring the scattered light at a scattering angle of $45^{\circ}$ as well as to measure the transmitted light by the means of a diode. Our sample is investigated at volume fractions of $1 \%$, $5 \%, 11.2 \%, 16 \%$, and $39.5 \%$ and at a temperature of $T$ $=14{ }^{\circ} \mathrm{C}$. The collected data does not only allow distinguishing between ergodic and nonergodic states but can also be analyzed for the internal dynamics of the system, as described in Secs. IV B 1 and IV B 2.

\section{Ergodic to nonergodic transition}

The transition from an ergodic to a nonergodic state is clearly observable when phase space averaged correlation functions of the system can be measured. This is easily done using the two-cell DWS technique, which was developed for this purpose. The normalized raw data for a volume fraction of $16 \%$ are shown exemplarily on the left side of Fig. 4. With increasing pressure, the measured correlation functions decay at larger lag times and eventually start to build up a plateau with a second decay, whereby the latter can easily be identified as the decay of the second cell. For better illustration, the decay of only the second cell is shown as well, which is separately measured by replacing the sample with a static scatterer such as a piece of Teflon or paper. The correlation functions of only the sample can be calculated using Eq. (12), by dividing the respective measured correlation functions by the second cell decay (when the system is still ergodic, the correlation functions can be measured without the use of the second cell). The resulting correlation functions are shown on the right side of Fig. 4. Clearly the correlation functions decay to zero for all pressures up to 1065 bars and start to exhibit a plateau at 1113 bars and above. The development of such a plateau is characteristic for a nonergodic behavior, whereas the decorrelation to zero is a sign of ergodicity. Thus, the ergodic to nonergodic transition for a volume fraction of $16 \%$ takes place at a pressure between 1065 and 1113 bars. Although the two-cell technique limits the range of detectable correlation times for nonergodic systems, it is nevertheless possible to clearly distinguish ergodic and arrested states. Furthermore, if only qualitative information (i.e., the nonergodic transition) is of interest, also samples with turbidity well below the DWS regime can be characterized since the second cell acts only as a device which averages the scattered light in phase space.

\section{Internal dynamics}

The correlation functions as shown above in the right part of Fig. 4 contain much more information than only the possibility to distinguish ergodic and nonergodic states. Corrected properly, they can be used for a quantitative analysis of the samples internal dynamics if the sample is turbid enough to apply the DWS theory (approximately $l^{*} \geq 5 L$ ). In our case, only a relatively small part of the investigated phase diagram allows for the application of the DWS theory. So, in order to discuss all our measurements, we restrict ourselves to a qualitative analysis of the sample dynamics. Therefore, we fit the decay of the correlation functions with the cumulant expansion (as described in Sec. III B). The first cumulant $\Gamma$ is a characteristic quantity describing the dynamics of the sample and is strongly influenced by the multiple scattering in the sample. To correct for the multiple scattering, we divide $\Gamma$ by the characteristic number of randomwalk steps $n_{c} \approx\left(L / l^{*}\right)^{2}$ of the photon on its way through the sample and get the multiple scattering corrected coefficient $\Gamma_{\text {corr }}$ which represents the dynamics of one scattering particle of the sample. This correction is strictly only valid when the photon trajectory in the sample can be described using a diffusion approach, as it is used in the DWS theory [it there- 


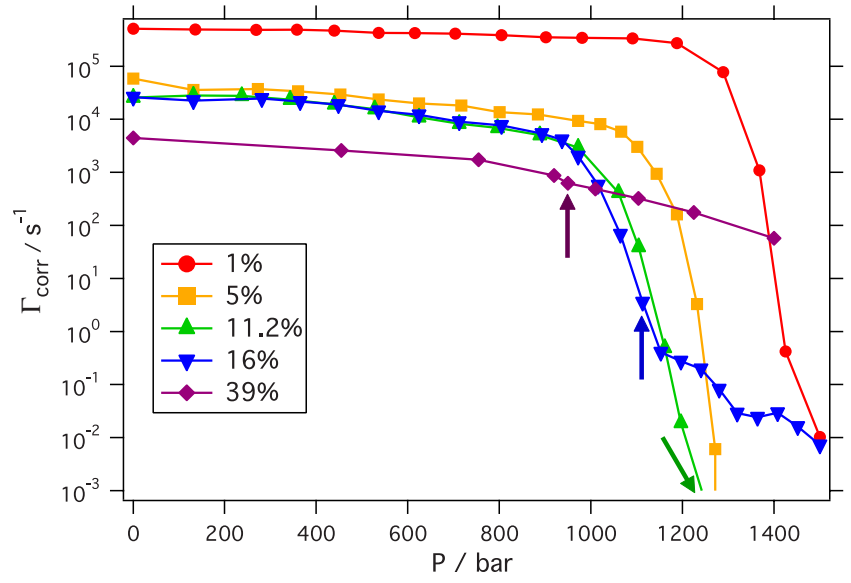

FIG. 5. (Color online) The cumulant fit coefficient $\Gamma_{\text {corr }}$ is corrected for multiple scattering and plotted against pressure at a temperature of measurement of $T=14{ }^{\circ} \mathrm{C}$. The arrows indicate the first nonergodic file measured as described in Sec. III C. The samples with a volume fractions of $1 \%$ and $5 \%$ remain ergodic.

fore also appears in Eq. (11)]. However we find it reasonable to correct all our data points for multiple scattering using this approach since the interesting part of the analysis fulfils the condition of a diffusing photon transport or is very close to it. The corrections for the points relatively far away from this condition are small and the values stay nearly constant in that region of the phase diagram, so they illustrate that the system is not changing drastically and therefore mainly have a function as a guide to the eye.

The parameters needed for the calculation of $\Gamma_{\text {corr }}$ are easily accessible: while the sample thickness $L$ is a given constant, the transport mean free path $l^{*}$ depends on the optical properties of the sample and varies strongly during the experiment. For ambient pressure, $l^{*}$ is calculated using Mie theory and compared to calibration samples via transmission measurements. Since $L / l^{*}$ is inversely proportional to the sample transmission (in the case of strong multiple scattering), the value of $l^{*}$ for all pressures can easily be calculated using the transmission values measured with our setup. The values of $\Gamma_{\text {corr }}$ calculated in such a manner are plotted against pressure in Fig. 5.

The sharp decrease of $\Gamma_{\text {corr }}$ for the samples with volume fractions $1 \%, 5 \%$, and $11.2 \%$ can be interpreted as phase separation, where the growth of the different phases is reflected in the slowing down of the dynamics of the scattered light. Like expected, the 39\% sample does not show such a steep decrease, while the $16 \%$ sample combines both trends. Indicating the transition from ergodic to nonergodic behavior (measured as described in Sec. III B) in the form of arrows to the graph, we can clearly see that the percolation can either drastically slow down the phase separation as for $16 \%$ or completely prevent it as for $39 \%$. This result is completely in agreement with the phase diagram from the simple test tube experiments ${ }^{18}$ and nicely shows that the percolation line crosses the phase separation line, effectively hindering the phase separation.

\section{Global fit of SANS data}

Using the global fit formalism, as shortly described in Sec. III D, and more comprehensively described in our pre-
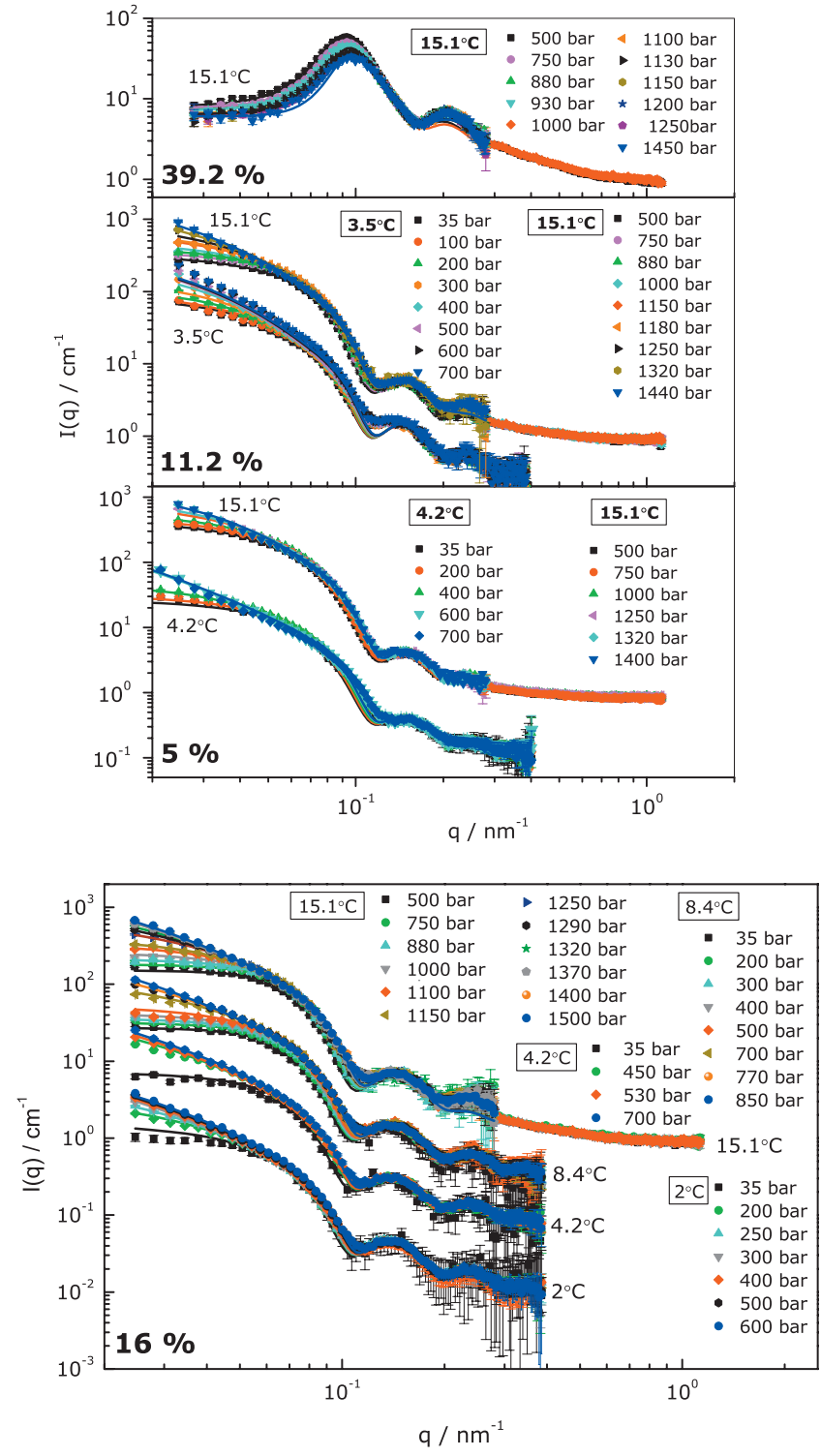

FIG. 6. (Color) $I(q)$ vs $q$ for different volume fractions (top: $5 \%, 11.2 \%$, and $39.2 \%$; bottom: 16\%) and temperatures as given in the figures (plotted with an offset). In each data set the pressure is varied and values are indicated in the figures. Full lines are calculated according to Eq. (13) with $P(q)$ according to Ref. 18. The $S(q)$ was calculated on the basis of the Robertus model with sticky hard sphere interactions. Deviations of fit from data at intermediate $q$ are due to not taking the experimental resolution into account. This was proven not to influence the analysis of the stickiness at small $q$. For details of fit see text.

vious paper, ${ }^{18}$ but now including stickiness as described in Sec. II, we were able to describe all measured SANS curves for all volume fractions, temperatures and pressure in the homogenous phase. The result is shown in Fig. 6. We have calculated $I(q)$ along Eq. (13) thereby fixing the parameters for the form factor $P(q)$, which we know already from our previous study: ${ }^{18}$ the radius $R=34.2 \mathrm{~nm}$, the thickness of the shell $\Delta R=2.3 \mathrm{~nm}$ and the width of the size distribution $\sigma$ $=0.124 . S(q)$ is described by the Robertus model of a polydisperse sticky hard sphere interaction, cf. Eqs. (1)-(4). Using these equations in the global fit routine means that we have to fix some parameters, especially the value for $d \Theta / d P=1 / 77.5 \mathrm{kbar}^{-1}$, which was determined using DLS, cf. Sec. IV A. Further parameters being fixed are $P_{0}=1$ bar 
and $\Delta=0.5 \mathrm{~nm}$. The value for $\Delta$ is taken from De Kruif and Schouten. ${ }^{30}$ However, also a value of $\Delta=0.3 \mathrm{~nm}$ has been reported. ${ }^{24}$ There it is claimed that the fit results for $L$ and $\Theta$ do not change significantly although $\Delta$ differs by a factor of two. In the global fit routine, the scattering length densities have to be corrected for pressure dependence due to compressibility. In order to do so, the particle's core compressibility was estimated by a contrast variation experiment under high pressure; ${ }^{33}$ it was found to be in the order of 2 $\times 10^{-5}$ bar $^{-1}$, thus the particle is almost incompressible. Furthermore, the pressure dependence of the volume fraction, the number densities and the temperature dependencies of the scattering length densities (thermal expansion) were also taken into account. Finally, the following parameters have been determined from the global fit: $L=107$ and $\Theta_{0}$ $=282 \mathrm{~K}$. Verduin and Dhont ${ }^{24}$ report for a similar system in benzene $L=112$ and $\Theta_{0}=304 \mathrm{~K}$.

We are interested to obtain the percolation threshold from our global fit, in addition to the DWS data, and correlate it with the stickiness parameter $\tau_{B}(P, T)$. In this fit routine, $\tau_{B}(P, T)$ is related to the square well potential using

$\tau_{B}(P, T)= \begin{cases}\tau_{B}(P, T) & \text { for } \tau_{B}(P, T)>\tau_{B, \text { perc }}(\text { vol } \%) \\ \tau_{B, \text { perc }}(\text { vol } \%) & \text { otherwise },\end{cases}$

with

$\tau_{B}(P, T)=\frac{2 R+\Delta}{12 \Delta} \exp \left[-L\left(\frac{\Theta(P)}{T}-1\right)\right]$.

We assumed here that the scattering curve will not change anymore with pressure as soon as the percolation line is reached. The percolation occurs for a specific stickiness $\tau_{B \text {,perc }}($ vol $\%)$, which depends only on the volume fraction. Therefore the stickiness parameter, determined by SANS, stays constant for pressures above the percolation transition. We found from our global fit: $\tau_{B \text {,perc }}(39.2 \%)=3.05$, $\tau_{B, \text { perc }}(16 \%)=0.131, \tau_{B, \text { perc }}(11.2 \%)=0.122$, and $\tau_{B \text {,perc }}(5 \%)$ $=0.08$. It turns out that for the $5 \%$ sample we may have not reached the percolation line, hence we had to fix the value for $\tau_{B \text {,perc }}$. This finding is in agreement with the theoretical phase diagram shown in Fig. 2.

On the basis of these results a plot of the inverse stickiness versus pressure for the different temperatures of measurements can be calculated. We rather have plotted $1 / \tau_{B}$ because then the intersections with the percolation value are better visible. It is shown in Fig. 7. We will use these results together with all other data on the loci of transition lines in Sec. IV when we discuss the phase diagram in comparison with theoretical predictions.

\section{Critical scaling law}

From an inspection of the data shown in Fig. 6, it seems evident that the pressure mainly affects the forward scattering properties of the system. To analyze the small $q$ behavior we have performed a Guinier expansion to obtain $I(q=0)$ $=I_{0}$

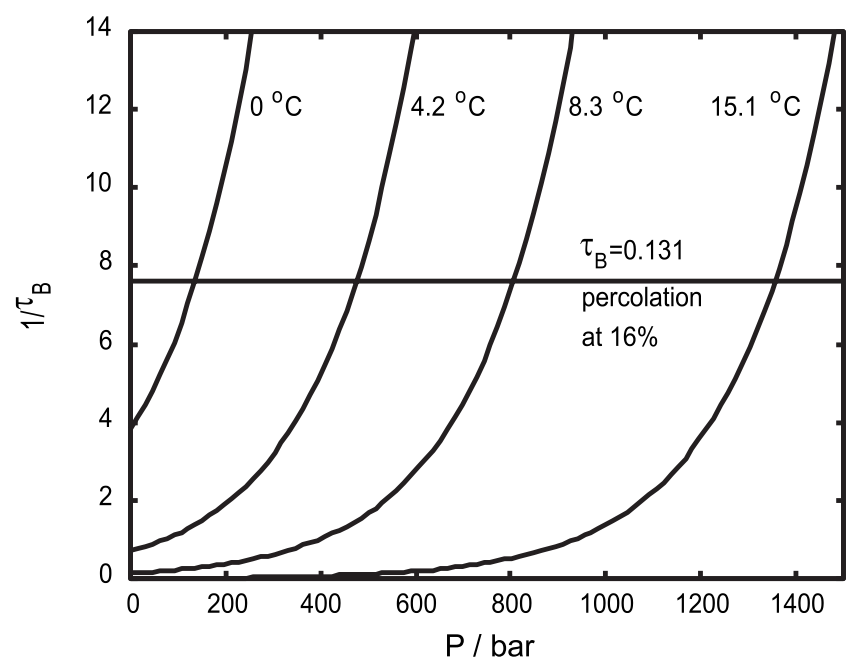

FIG. 7. Plot of inverse stickiness vs pressure calculated from parameters of the global fit for volume fraction $16 \%$ using Eq. (6).

$$
\ln I(q) \cong \ln I(q=0)-\frac{R_{g}^{2} q^{2}}{3},
$$

where $R_{g}$ is the radius of gyration. Using $I_{0}$ and the transition pressures at a given $T$, we can go a step further and use the notation well known in the physics of critical phenomena ${ }^{49}$ that the intensity scattered in forward direction diverges upon approaching the critical point by variation of temperature as $I_{0} \propto\left(\Delta T / T_{c}\right)^{-\gamma}$, with $\Delta T=\left|T-T_{c}\right|$ and $\gamma$ being the critical exponent for the susceptibility. We can formulate a similar expression for the $\Delta P=\left|P-P_{c}\right|$ behavior of the inverse intensity, thereby introducing the new reduced variable $\varepsilon$ $=\left(\Delta P / P_{c}\right)$

$$
I_{0} \propto\left(\frac{\Delta P}{P_{c}}\right)^{-\gamma} .
$$

Some remarks concerning the choice of $P_{c}$ are important to make. Most of our data are taken for the $16 \%$ sample. The respective transition pressure is the spinodal pressure, which we can read from Fig. 3(b) for a temperature of $T=15.1^{\circ} \mathrm{C}$. This value is $P_{c}=1364$ bar. Then a plot of $\varepsilon=\left(\Delta P / P_{c}\right)$ versus the intensity in a double log plot shown in Fig. 8 should result in a linear relation.

We find that the data are consistent with a mean field exponent of $\gamma=-1$, which is expected for the temperature variation of the susceptibility in liquid-liquid phase transitions of polymers belonging to the $\mathrm{H} 2$ group according to the Hohenberg-Halperin classification. ${ }^{49}$ A similar mean field type of behavior had also been observed by Lenstra and Dhont $^{50}$ in sheared colloidal systems. However, adhesive hard sphere systems are usually considered to be model systems for short range order thus exhibiting three-dimensional (3D) Ising-like critical behavior. Our data are not consistent with this assumption.

All data shown in Fig. 8 are taken in the homogenous regime before transient effects show up. The percolation threshold, as determined via DWS, would lie at a reduced pressure of about $\log \varepsilon=-0.95$. If we nevertheless calculate data points in the transient regime then these points would not follow the observed straight line behavior. How to treat 


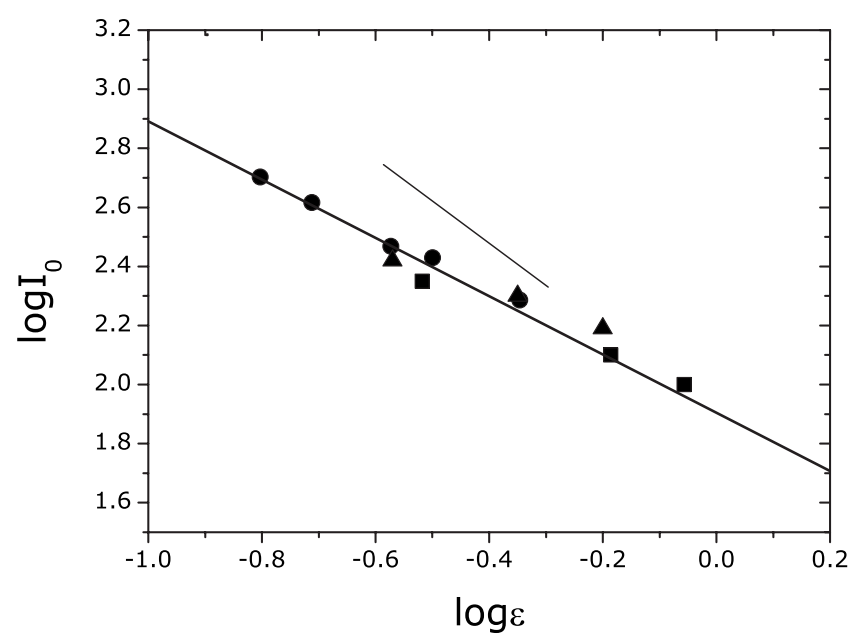

FIG. 8. The forward intensity plotted vs the reduced pressure according to Eq. (16). We used $P_{c}=1364$ bars for the $\varphi=0.16$ SANS data (filled circles) and LS (filled triangles) at $T=15.1^{\circ} \mathrm{C}$. SANS data (filled squares) at $T$ $=0{ }^{\circ} \mathrm{C}$ and $\varphi=0.112$ with $P_{c}=287$ bars [pressure value from Fig. 3(b) ]. The straight line through the data points has the slope of -1 suggesting a mean field type of behavior. All curves are vertically shifted to the $15.1{ }^{\circ} \mathrm{C}$ SANS data to show the general critical behavior irrespective of the chosen temperature of measurement. The other, steeper straight line shown in the figure has a slope of -1.24 , which would correspond to a scaling behavior of the 3D Ising case. Clearly, our data are not in agreement with this expectation.

critical scaling behavior in the vicinity of percolation effects is still an open question. The intensities $I_{0}$ used in Fig. 8 were obtained via Eq. (15), however, without any correction for the change in intensity due to the change in contrast through the changes in pressure. A rough estimation of the expected change in forward intensity using a $\Delta P$ of about 350 bars (between $\log \varepsilon=-0.4$ and -0.8 ) on the basis of the density changes of toluene ${ }^{33}$ at the given contrast of measurement for the $16 \%$ sample gives a $20 \%$ change in $I_{0}$. In Fig. 8 this corresponds to an upturn of the points for large negative $\log \varepsilon$-values, however, the mean field assignment is still valid.

Another possibility of rationalizing the type of critical scaling behavior stems from the analysis of the dynamic measurements depicted in Fig. 5. According to theory, ${ }^{51}$ the decay rate $\Gamma(q)$ is given by

$$
\Gamma(q)=\frac{L(q)}{S(q)} q^{2},
$$

where $L(q)$ is the Onsager coefficient being related to the short time diffusion behavior of the colloids and $S(q)$ is the structure factor. Usually, as $T$ approaches the critical temperature or, as in our case, the pressure $P$ approaches the critical pressure, the structure factor diverges and hence the rate $\Gamma(q)$ goes to zero. This feature is commonly known as critical slowing down. The scaling function for the rate is simply given by inserting the scaling function for the intensity, Eq. (16), for $S(q)$ into Eq. (17). Then for pressures not too close to the critical pressures a scaling relation with the mean field exponent $\gamma=1$ should emerge. ${ }^{52}$ This is shown in Fig. 9 using the DLS data shown in Fig. 5 and holds true for the $5 \%$ sample. The other two higher volume fractions show larger slopes.

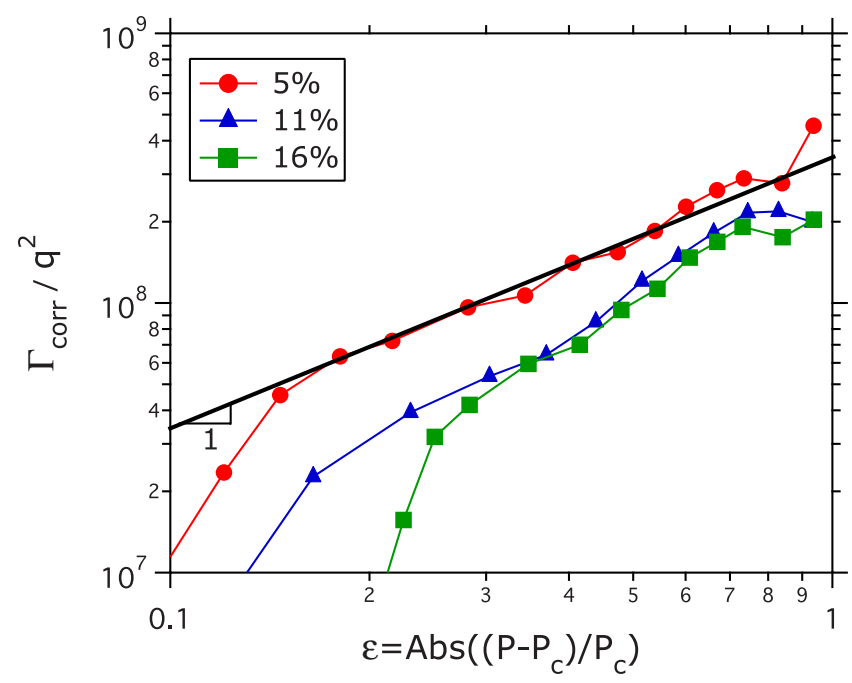

FIG. 9. (Color online) The relaxation rates divided by $q^{2}$ vs the reduced pressure for the 5,11 , and $16 \%$ samples at $T=15.1{ }^{\circ} \mathrm{C}$. The straight line has the slope of 1 suggesting mean field type of behavior. We have used the value for the critical pressure of $P_{c}=1364$ bars from $\varphi=0.1$, as deduced from Fig. 3(b). The same $P_{c}$ was used in Fig. 8. From the measured transmissions of our samples we have then determined the transmission at the $\varphi=0.11$ sample at that pressure and have used this value to determine the $P_{c}$ for the $\varphi=0.05$ sample.

For data in the proximity of the critical point, i.e., $q \xi$ $\cong 1$, the $q^{2}$ scaling has to be replaced by a $\Gamma \sim q^{\alpha}$ scaling, where $\alpha$ goes from $2 \rightarrow 3$ for $P \rightarrow P_{c}$. This comes from the fact that in the Stokes-Einstein relation, upon approaching the critical point, the radius of the particle is replaced by the only relevant length scale close to criticality, namely, the correlation length $\xi$, which is proportional to $q^{-1}$. Then the above given proportionality between $\Gamma$ and $q$ emerges. ${ }^{53}$

However, we have no possibility to take $q$-dependent data with DLS under high pressures yet. A multiangle high pressure cell to overcome this deficiency is under construction. We will address this issue in future work to test our hypothesis.

Also we find deviations at small $\varepsilon$, which are due to the proximity of the percolation threshold in a similar fashion as already argued in connection with Fig. 8.

In the foregoing sentences we have mentioned the correlation length $\xi$, which can be obtained from an OrnsteinZernike approach to the $q$-dependent SANS data as the square root of the slope in a plot $I(q=0) / I(q)$ versus $q^{2}$. A tentative analysis of the $16 \%$ SANS data yields a mean field type of behavior and a value of $\xi \cong 30 \mathrm{~nm}$ for $\varepsilon \cong 0.15$. In analogy to a polymer blend system we expect a cross over to $3 \mathrm{D}$ Ising behavior at about that $\varepsilon$ value at which the Debye cutoff, marking the onset of the mode coupling corrections, is in the order of the particle size. ${ }^{52}$

A possible argument why instead of the entirely expected Ising scaling, typical for systems with short range interactions, we find a mean field type of behavior lies in the polydispersity of the system. It acts similar as an extended structure and increases virtually the interaction range. This situation is then reminiscent to a polymeric system, which also shows mean field type critical behavior. ${ }^{52}$ Although Miller and Frenkel $^{7}$ have explicitly found Ising scaling in 


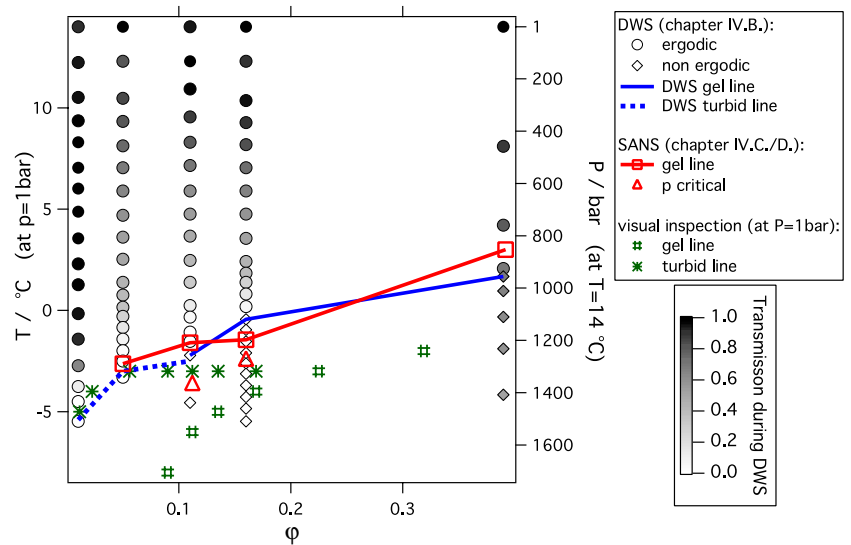

FIG. 10. (Color online) Final experimental phase diagram. Lines are guides to the eye. Dotted line: DWS coexistence line. Solid line: DWS percolation line. Dashed line: SANS percolation line. The phrase "visual inspection" in the inset refers to the phase diagram given in Ref. 18. All other symbols are explained in the inset.

their paper, we should note that they have performed the calculations for a monodisperse case, which is not applicable here.

\section{E. Phase diagram}

Our principal goal was to construct a phase diagram of a polydisperse sticky hard sphere system based upon experimental techniques which have not been used so far (pressure techniques and DWS). Our knowledge is mainly based on two different kinds of experimental tools, namely SANS and DWS, where both techniques were applied under high pressure conditions. Putting all data together we find our experimentally determined phase diagram. It is shown in Fig. 10, referenced to $T=14^{\circ} \mathrm{C}$. Obviously, we find a difference in the locus of our DWS gel line with the gel line from visual observation of a tilted test tube. ${ }^{18}$ This is not surprising as the gel line in the phase diagram obtained by visual inspection corresponds to a gel that resists to flow on applying a finite force or stress, whereas the DWS percolation threshold corresponds to a (gel) structure which is probably not able to resist a finite force. Indeed, the position of the gel line de- pends on the force or stress applied to the system as shown by Trappe et al. ${ }^{11}$ Or to cite these authors explicitly: "In general, a sufficiently large stress will cause a jammed solid sample to yield and flow; thus the yield stress $\sigma_{y}$ defines the phase boundary." Therefore, DWS and SANS percolation can be compared to theoretical and simulation results, whereas the gelation observed by tilting the sample is found at lower $\tau_{B}$ as expected (the gel line is shifted to lower $\tau_{B}$ but exhibits the same shape). Our percolation results are compared to theoretical, ${ }^{21} \mathrm{cf}$. Eqs. (7) and (8), and simulation results $^{7,35}$ in Fig. 11 showing excellent agreement. We have measured that for the first time via DWS, which gives access to the ergodic to nonergodic transition directly.

On the other hand the data for the coexistence line are found to lie rather consistent within the old visually inspected phase diagram, see Fig. 10. We have tried to find a criterion for the respective transition pressures and thus temperatures on the basis of equal transmissions obtained at different volume fractions. Specifically, during the DWS measurements, the intensity of the transmitted light was permanently recorded. Since the phase separation of the sample is reflected in increased turbidity, the turbid line can, in principle, be deduced from the transmission values. To do so, the measurements of $d P / d T_{\text {trans }}$ in Sec. IV A were used to estimate a correct value for phase separation. From Fig. 3(b) we deduce $P_{c}=1364$ bars for the sample with $\varphi=0.10$ at $T$ $=15.1{ }^{\circ} \mathrm{C}$. The variation of $P_{c}$ is negligible at volume fractions around 0.10 (according to visual inspection), and therefore it is reasonable to assume the same $P_{c}$ for a sample with $\varphi=0.11$ at the same temperature. The transmission value at this point then defines the coexistence line for all samples during the DWS measurements, shown as a dotted line in Fig. 10 (converted to the according temperature for a constant pressure of 1 bar). It nicely overlays with the results from the visual inspection except for the case of $\varphi=0.16$. This is not surprising since at this temperature, the sample is already deep in the nonergodic regime, where all dynamic processes are arrested at short length scales (see Fig. 5). We have therefore added a differently measured data point ( $p$ critical) for the $\varphi=0.16$ sample, which confirms the expected

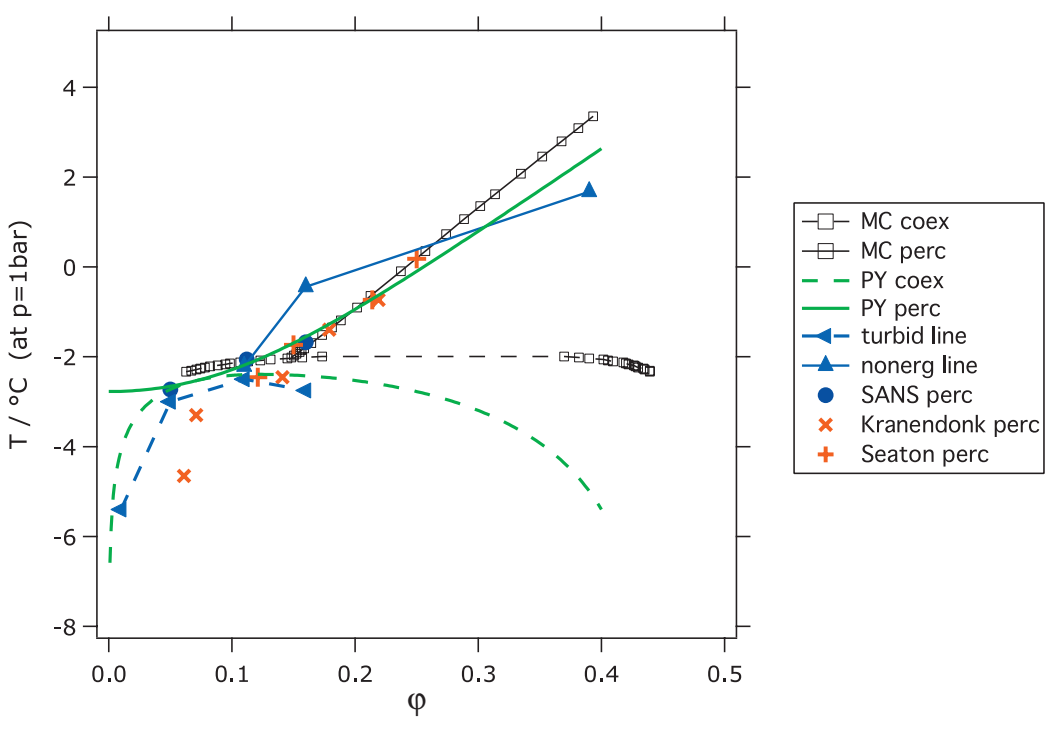

FIG. 11. (Color) Comparison between theoretical phase diagram and our data (triangles). Data are converted into temperature using our global fit parameters and Eq. (6). Also shown is simulated percolation data from Kranendonk et al. (Ref. 6) and Seaton and Glandt (Ref. 5). MC is data from Miller and Frenkel (Ref. 7) which is similar to the data of Fantoni et al. (Ref. 35, see Fig. 2). The PY model is given by Eqs. (7) and (8), respectively. 
behavior of a rather flat coexistence curve for not too high values of $\varphi$, see Figs. 2 and 11. The problem on how to deduce phase coexistence under the action of percolation from our (DWS, DLS) transmission data is still open to debate.

Our next concern is the influence of percolation on the demixing by performing transient experiments. Without going into detail concerning that issue, which will be a subject of a pending publication, ${ }^{54}$ we show in Fig. 10 only data in the final stage, after all transient effects have relaxed. We believe that this is possible without affecting our argumentation. With regard to metastable and nonstable no clear differentiation could be made, except that we know from our DLS data that binodal and spinodal are very close to each other in the order of $1 \mathrm{~K}$, see Fig. 3(b).

We observe almost no differences between theoretical and experimentally observed phase diagram as shown in Fig. 11 , based upon the experimental parameters from the global fit applying the formalism from Sec. IV C. However, if we would have let the global fit be free in all parameters, then for $d P / d T_{\text {trans }}$ a value of $67 \mathrm{bar} / \mathrm{K}$ would come out. This, in turn, would shift the experimental phase diagram of $2.5 \mathrm{~K}$ higher. That on the other hand corresponds to roughly a factor of 2 in $\tau_{B}$ as calculated from Eq. (6). This seemingly large difference is put into perspective through a rather large number of different values found for $\tau_{B}$ with respect to percolation and coexistence depending on which model is used and which approximation is chosen. However, what always agrees is the form of the curves. What does this mean? Obviously the details of the potential influence the exact loci of lines in Fig. 11 and specifically the degree of overlap between adjacent spheres, which allows shifting the curves vertically. Hence our agreement might be fortuitous. Our results support the simulations by Miller and Frenkel ${ }^{7}$ and by Fantoni et al. ${ }^{35}$ at least with respect to the percolation limit. The value for the critical volume fraction from their simulation is, however, difficult to verify with our experiments. The reason is the proximity between binodal and spinodal curves in the volume fraction range studied which makes it practically impossible to differentiate between spinodal decomposition and phase separation via a nucleation and growth mechanism. However, please note that the scaling properties refer for theoretical reasons to the spinodal. For our purpose taking the volume fraction range we studied into account, we find that the $\mathrm{C} 1$ model taken from the paper by Fantoni et $a l^{35}$ is in close agreement with Penders and Vrij ${ }^{21}$ and thus the approximate equations for the phase diagram as given by Eqs. (7) and (8). From an experimental point of view it is not possible to distinguish between the various approximations.

\section{CONCLUSIONS}

We presented in this paper a combination of light scattering techniques (DLS and DWS) with SANS under high pressure. The data from the latter were analyzed using a global fit routine with a model taking into account the structure factor of a polydisperse sticky hard sphere system using the Robertus model. ${ }^{32}$ The parameters obtained from this fit routine can be utilized to predict the phase diagram of a polydisperse sticky hard sphere colloidal system including the percolation transition. We have confirmed this ergodic to nonergodic transition by DWS under high pressures. Pressure was also used for the first time as the leading variable for the dynamic measurements, which facilitated probing the phase diagram. From dynamic light scattering experiments characteristic signatures for binodal and spinodal points could be found. The agreement between our experimentally determined phase diagram with theoretical ones is good concerning the absolute values for the stickiness and shape. By using the Robertus model, our data provide for the first time a full account of the realistically modeled scattering curves including polydispersity. Other theoretical models assume monodisperse systems using a simplified expression for the structure factor of which we know that it gives wrong results at low $q \cdot{ }^{18}$ Furthermore, polydispersity is likely the reason why we find a mean field type of critical scaling behavior contrary to the expectation and a calculation by Miller and Frenkel $^{7}$ suggesting 3D Ising scaling.

\section{ACKNOWLEDGMENTS}

The SANS data are based on experiments performed at the Swiss spallation neutron source SINQ, Paul Scherrer Institute, Villigen, Switzerland. We thank A. Giacometti for sending numerical data for Fig. 2. E. Zaccarelli, A. Giacometti, and Jan Dhont are thanked for stimulating discussions. A. Wilk and M. Ratajczyk acknowledge support within the European Network of Excellence SoftComp.

${ }^{1}$ N. A. M. Verhaegh, D. Asnaghi, H. N. W. Lekkerkerker, M. Giglio, and L. Cipelletti, Physica A 242, 104 (1997).

${ }^{2}$ R. O. Watts, D. Henderson, and R. J. Baxter, Adv. Chem. Phys. 21, 422 (1971).

${ }^{3}$ Y. C. Chiew and E. D. Glandt, J. Phys. A 16, 2599 (1983).

${ }^{4}$ S. A. Safran, I. Webman, and G. S. Grest, Phys. Rev. A 32, 506 (1985).

${ }^{5}$ N. A. Seaton and E. D. Glandt, J. Chem. Phys. 86, 4668 (1987).

${ }^{6}$ W. Kranendonk and D. Frenkel, Mol. Phys. 64, 403 (1988).

${ }^{7}$ M. A. Miller and D. Frenkel, Phys. Rev. Lett. 90, 135702 (2003); M. A. Miller and D. Frenkel, J. Chem. Phys. 121, 535 (2004).

${ }^{8}$ C. Patrick Royall, S. R. Williams, T. Ohtsuka, and H. Tanaka, Nature Mater. 7, 556 (2008).

${ }^{9}$ Y. Gao and M. L. Kilfoil, Phys. Rev. Lett. 99, 078301 (2007).

${ }^{10}$ C. J. Dibble, M. Kogan, and M. J. Solomon, Phys. Rev. E 77, 050401(R) (2008).

${ }^{11}$ V. Trappe, V. Prasad, L. Cipelletti, P. N. Segrè, and D. A. Weitz, Nature (London) 411, 772 (2001).

${ }^{12}$ F. Cardinaux, T. Gibaud, A. Stradner, and P. Schurtenberger, Phys. Rev. Lett. 99, 118301 (2007).

${ }^{13}$ S. Manley, H. M. Wyss, K. Miyazaki, J. C. Conrad, V. Trappe, L. J. Kaufman, D. R. Reichman, and D. A. Weitz, Phys. Rev. Lett. 95, 238302 (2005).

${ }^{14}$ P. N. Segrè, V. Prasad, A. B. Schofield, and D. A. Weitz, Phys. Rev. Lett. 86, 6042 (2001).

${ }^{15}$ C. Haro-Pérez, L. F. Rojas-Ochoa, R. Castañeda-Priego, M. Quesada-Pérez, J. Callejas-Fernández, R. Hidalgo-Álvarez, and V. Trappe, Phys. Rev. Lett. 102, 018301 (2009).

${ }^{16}$ K. N. Pham, A. M. Puertas, J. Bergenholtz, S. U. Egelhaaf, A. Moussaïd, P. N. Pusey, A. B. Schofield, M. E. Cates, M. Fuchs, and W. C. K. Poon, Science 296, 104 (2002).

${ }^{17}$ S. F. Edwards and K. E. Evans, J. Chem. Soc., Faraday Trans. II 78, 113 (1982). 
${ }^{18}$ J. Kohlbrecher, J. Buitenhuis, G. Meier, and M. P. Lettinga, J. Chem. Phys. 125, 044715 (2006).

${ }^{19}$ J. W. Jansen, C. G. de Kruif, and A. Vrij, J. Colloid Interface Sci. 114, 481 (1986).

${ }^{20}$ P. W. Rouw and C. G. de Kruif, J. Chem. Phys. 88, 7799 (1988).

${ }^{21}$ M. H. G. M. Penders and A. Vrij, Adv. Colloid Interface Sci. 36, 185 (1991).

${ }^{22}$ A. T. J. M. Woutersen and C. G. de Kruif, J. Chem. Phys. 94, 5739 (1991).

${ }^{23}$ M. H. G. M. Penders, A. Vrij, and R. van der Haegen, J. Colloid Interface Sci. 144, 86 (1991)

${ }^{24}$ H. Verduin and J. K. G. Dhont, J. Colloid Interface Sci. 172, 425 (1995)

${ }^{25}$ M. C. Grant and W. B. Russel, Phys. Rev. E 47, 2606 (1993).

${ }^{26}$ A. T. J. M. Woutersen, J. Mellema, C. Blom, and C. G. de Kruif, J. Chem. Phys. 101, 542 (1994).

${ }^{27}$ S. Roke, O. Berg, J. Buitenhuis, A. van Blaaderen, and M. Bonn, Proc. Natl. Acad. Sci. U.S.A. 103, 13310 (2006).

${ }^{28}$ S. Roke, J. Buitenhuis, J. C. van Miltenburg, M. Bonn, and A. van Blaaderen, J. Phys.: Condens. Matter 17, S3469 (2005).

${ }^{29}$ For a review especially on colloidal gels, see E. Zaccarelli, J. Phys.: Condens. Matter 19, 323101 (2007).

${ }^{30}$ C. G. De Kruif and J. A. Schouten, J. Chem. Phys. 92, 6098 (1990).

${ }^{31}$ R. J. Baxter, J. Chem. Phys. 49, 2770 (1968).

${ }^{32}$ C. Robertus, W. H. Philipse, J. G. H. Joosten, and Y. K. Levine, J. Chem. Phys. 90, 4482 (1989).

${ }^{33}$ G. Meier, R. Vavrin, J. Kohlbrecher, J. Buitenhuis, M. P. Lettinga, and M. Ratajczyk, Meas. Sci. Technol. 19, 034017 (2008).

${ }^{34}$ M. Beiner, G. Fytas, G. Meier, and S. Kumar, J. Chem. Phys. 116, 1185 (2002); S. Janssen, D. Schwahn, K. Mortensen, and T. Springer, Macromolecules 26, 5587 (1993).

${ }^{35}$ R. Fantoni, D. Gazzillo, and A. Giacometti, J. Chem. Phys. 122, 034901 (2005)
${ }^{36}$ S. Buzzaccaro, R. Rusconi, and R. Piazza, Phys. Rev. Lett. 99, 098301 (2007).

${ }^{37}$ P. J. Lu, E. Zaccarelli, F. Ciulla, A. B. Schofield, F. Sciortino, and D. A. Weitz, Nature (London) 453, 499 (2008).

${ }^{38}$ S. Eckert, G. Meier, and I. Alig, Phys. Chem. Chem. Phys. 4, 3743 (2002).

${ }^{39}$ W. Stöber, A. Fink, and E. Bohn, J. Colloid Interface Sci. 26, 62 (1968).

${ }^{40}$ A. K. van Helden, J. W. Jansen, and A. Vrij, J. Colloid Interface Sci. 81, 354 (1981).

${ }^{41}$ J. Kohlbrecher, A. Bollhalder, R. Vavrin, and G. Meier, Rev. Sci. Instrum. 78, 125101 (2007).

${ }^{42}$ G. Meier and H. Kriegs, Rev. Sci. Instrum. 79, 013102 (2008).

${ }^{43}$ B. J. Berne and R. Pecora, Dynamic Light Scattering (Wiley, New York, 1976)

${ }^{44}$ P. Stepanek, in Dynamic Light Scattering, edited by W. Brown (Clarendon, Oxford, 1993).

${ }^{45}$ D. A. Weitz and D. J. Pine, Diffusing Wave Spectroscopy (Oxford University Press, Oxford, 1993).

${ }^{46}$ S. Romer, F. Scheffold, and P. Schurtenberger, Phys. Rev. Lett. 85, 4980 (2000).

${ }^{47}$ U. Keiderling, Appl. Phys. A: Mater. Sci. Process. 74, S1455 (2002).

${ }^{48}$ J. S. Higgins and H. C. Benoit, Polymers and Neutron Scattering (Oxford Science, Oxford, 1994).

${ }^{49}$ H. E. Stanley, Phase Transitions and Critical Phenomena (Clarendon, Oxford, 1971).

${ }^{50}$ T. A. J. Lenstra and J. K. G. Dhont, Phys. Rev. E 63, 061401 (2001).

${ }^{51}$ H. Swinney and D. Henry, Phys. Rev. A 8, 2586 (1973).

${ }^{52}$ G. Meier, B. Momper, and E. W. Fischer, J. Chem. Phys. 97, 5884 (1992).

${ }^{53}$ K. Perl and R. Ferrell, Phys. Rev. Lett. 29, 51 (1972).

${ }^{54}$ J. Buitenhuis, J. Kohlbrecher, M. P. Lettinga, G. Meier, M. Ratajczyk, K. Tomczyk, R. Vavrin, and A. Wilk (unpublished). 